\title{
Gauge Invariance of Gravitodynamical Potentials in the Jefimenko's Generalized Theory of Gravitation
}

\author{
Augusto Espinoza, Andrew Chubykalo, David Perez Carlos \\ Unidad Académica de Física, Universidad Autónoma de Zacatecas, Zacatecas, México \\ Email: achubykalo@yahoo.com.mx
}

How to cite this paper: Espinoza, A., Chubykalo, A. and Carlos, D.P. (2016) Gauge Invariance of Gravitodynamical Potentials in the Jefimenko's Generalized Theory of Gravitation. Journal of Modern Physics, 7, 1617-1626.

http://dx.doi.org/10.4236/jmp.2016.713146

Received: July 5, 2016

Accepted: September 3, 2016

Published: September 6, 2016

Copyright $\odot 2016$ by authors and Scientific Research Publishing Inc. This work is licensed under the Creative Commons Attribution International License (CC BY 4.0).

http://creativecommons.org/licenses/by/4.0/

(c) (i) Open Access

\begin{abstract}
In the Jefimenko's generalized theory of gravitation, it is proposed the existence of certain potentials to help us to calculate the gravitational and cogravitational fields, such potentials are also presumed non-invariant under certain gauge transformations. In return, we propose that there is a way to perform the calculation of certain potentials that can be derived without using some kind of gauge transformation, and to achieve this we apply the Helmholtz's theorem. This procedure leads to the conclusion that both gravitational and cogravitational fields propagate simultaneously in a delayed and in an instant manner. On the other hand, it is also concluded that these potentials thus obtained can be real physical quantities, unlike potentials obtained by Jefimenko, which are only used as a mathematical tool for calculating gravitational and cogravitational fields.
\end{abstract}

\section{Keywords}

Gravitational Potentials, Cogravitation, Helmholtz's Theorem

\section{Introduction}

Jefimenko's generalization of Newton's gravitational theory [1] [2] is based to a large extent on the assumption that there exists a second gravitational field (which he has named the cogravitational, or Heaviside's, field). Note that there are several publications in which it is suggested that a second field can be involved in gravitational interactions (see [3] and, e.g., [4] and references there). The first such publication was by Oliver Heaviside [3], unfortunately his article appears to have been generally ignored (see, e.g. [4] pp. 103-104). The overriding reason why Heaviside's work did not attract the attention was that his single article on gravitation was eventually completely eclipsed 
by Einstein's brilliant and spectacularly successful general relativity theory. It is noteworthy, however, that Newton's gravitational theory generalized to time-dependent systems yields several results which heretofore are believed the exclusive consequence of the general relativity theory. Jefimenko discusses this very important circumstance in [1] [2]. It is interesting to note that Einstein, four years before he published his general relativity theory, published an article on the possibility of a gravitational analogue of electromagnetic induction. Also this article was practically unknown, possibly because it was published in a rather inappropriate journal whose title (translated from the German) was: "Quarterly Journal for Forensic Medicine and Public Sanitation;" (see [5]).

Newton's theory does not include inductive phenomena, but a relativistic theory of gravitation should include them. Indeed, under the relativistic mass-energy equivalence, not only the mass is a source of gravitational field but any kind of energy also is. Therefore, a body creates gravitational field not only by mass but also by their kinetic energy, i.e. by their movement. And this, ultimately, is what it means induction: the production of forces by moving bodies [6].

In the general relativity theory, Einstein predicted the existence of gravitational induction phenomena, such phenomena are appointed by Einstein as gravitomagnetism. It can be showed that Jefimenko equations are also derived from linearized Einstein equations (see, for example, pp 47 and 48 in [6]).

Since in 2004, NASA has orbited the "Gravity Probe B", whose purpose was to prove the existence of gravitomagnetism, (see [7] [8]).

In order to describe the time-dependent gravitational systems, the Jefimenko's generalized theory of gravitation is based on postulating of retarded expressions for the accustomed gravitational field $\boldsymbol{g}$ and the Heaviside's or cogravitational field $\boldsymbol{K}$ (Heaviside [3] was the first who supposed the existence of this field making an analogy between gravitational and electromagnetic fields), where the field $\boldsymbol{g}$ acts to and arises from motionless as well as moving masses, and the field $K$ acts to and arises from exclusively moving masses. Let us from this point call this theory "gravitodynamics", and the complex of the fields $\boldsymbol{g}$ and $\boldsymbol{K}$ call the "gravitodynamical field". Jefimenko taking into account mentioned retarded expressions for $\boldsymbol{g}$ and $\boldsymbol{K}$, obtains the system of equations for which these expressions are solutions. These equations are analogous to Maxwell's equations. In principle, this method was proposed to eliminate the possibility of instantaneous solutions from the discussion. There against in this work we are going to postulate the system of differential equations rather than solutions for the gravitational dynamics, and we will obtain both retarded and instantaneous solutions for the fields $\boldsymbol{g}$ and $K$. The gravitational field $g$ behaves analogously to the electric field in the Maxwell's electromagnetic theory, and cogravitational field $K$ analogously to the magnetic field.

First of all, we write the equations describing time-dependent gravitational systems [1] (see p. 120) and [2]

$$
\begin{aligned}
& \nabla \cdot \boldsymbol{g}=-4 \pi G \varrho \\
& \nabla \cdot \boldsymbol{K}=0,
\end{aligned}
$$




$$
\begin{aligned}
& \nabla \times \boldsymbol{g}=-\frac{\partial \boldsymbol{K}}{\partial t}, \\
& \nabla \times \boldsymbol{K}=\frac{1}{c^{2}} \frac{\partial \boldsymbol{g}}{\partial t}-\frac{4 \pi G}{c^{2}} \boldsymbol{J},
\end{aligned}
$$

where $c$ is the velocity of propagation of the fields, which is supposed equal to the velocity of light for the retarded component, $G$ is the constant of gravitation, $\varrho$ is the density of mass and $\boldsymbol{J}=\varrho \boldsymbol{v}$ is the density of mass current.

There are some differences between Maxwell's equations of electrodynamics and the Jefimenko's equations of gravitation, i.e. the analogy is not perfect. For example, we have two kinds of electric charges, positives and negatives, which repel each other if the charges are equal and attract each other if they are different, whereas while we have only one type of mass, and if we have a system of two masses in repose, they always attract each other. While the electric field is directed from positive charges generating this field and is directed to the negative charges, the gravitational field is always directed to the masses by which is created. Another difference is that the magnetic field is always righthanded relative to the electric current by which is created, while the cogravitational field is always left-handed relative to the mass current by which is created.

In the analogy between electrodynamics and the so-called gravitodynamics, following the Jefimenko's book [1] we can resume the correspondence between electromagnetic and gravitodynamic symbols and constants in the following Table 1.

\section{The Gravitodynamical Potentials}

Here, we introduce as is made in electrodynamics, the gravitodynamical potentials. If

\begin{tabular}{|c|c|}
\hline Electromagnetic & Gravitational \\
\hline$q \quad$ (charge) & $m$ (mass) \\
\hline$\varrho \quad$ (volume charge density) & $\varrho$ (volume mass density) \\
\hline$\sigma$ (surface charge density) & $\sigma$ (surface mass density) \\
\hline$\lambda_{e}$ (line charge density) & $\lambda_{m}$ (line mass density) \\
\hline$\varphi \quad$ (electric's scalar potential) & $\tau$ (mass's scalar potenctial) \\
\hline A (magnetic vector potential) & $\Gamma$ (cogravitational vector potential) \\
\hline $\boldsymbol{J}_{e} \quad$ (convection current density) & $\boldsymbol{J}$ (mass-current density) \\
\hline$I_{e} \quad($ electric current $)$ & $I \quad$ (mass current) \\
\hline $\boldsymbol{m}$ (magnetic dipole moment) & $\boldsymbol{d}$ (cogravitational moment) \\
\hline $\boldsymbol{E}$ (electric field) & $g$ (gravitational field) \\
\hline $\boldsymbol{B}$ (magnetic field) & $K($ cogravitational field $)$ \\
\hline$\varepsilon_{0} \quad($ permittivity of space $)$ & $-1 / 4 \pi G$ \\
\hline$\mu_{o} \quad$ (permeability of space) & $-4 \pi G / c^{2}$ \\
\hline$-1 / 4 \pi \varepsilon_{0}=-\mu_{0} c^{2} / 4 \pi$ & $G$ (gravitational constant) \\
\hline
\end{tabular}

Table 1. Corresponding electromagnetic and gravitodynamic symbols and constants. 
the cogravitational field $K$ satisfies Equation (2), we can always write it as the curl of some other vector quantity $\Gamma$,

$$
\boldsymbol{K}=\nabla \times \Gamma
$$

where $\Gamma$ is the gravitodynamical vector potential. Substituting Equation (5) in (3), we obtain

$$
\nabla \times\left(\boldsymbol{g}+\frac{\partial \Gamma}{\partial t}\right)=0
$$

The quantity within the parentheses can be written as the gradient of a gravitodynamical scalar potential $\tau$ :

$$
\boldsymbol{g}+\frac{\partial \Gamma}{\partial t}=-\nabla \tau
$$

therefore,

$$
\boldsymbol{g}=-\nabla \tau-\frac{\partial \Gamma}{\partial t}
$$

Substituting the expressions (5) and (8) for the fields $\boldsymbol{g}$ and $\boldsymbol{K}$, in the inhomogeneous Equations (1) and (4), we obtain

$$
\nabla^{2} \tau+\frac{\partial}{\partial t}(\nabla \cdot \Gamma)=4 \pi G \varrho,
$$

and

$$
\nabla^{2} \Gamma-\frac{1}{c^{2}} \frac{\partial^{2} \Gamma}{\partial t^{2}}-\nabla\left(\nabla \cdot \Gamma+\frac{1}{c^{2}} \frac{\partial \tau}{\partial t}\right)=\frac{4 \pi G}{c^{2}} \boldsymbol{J} .
$$

Equations (9) and (10) can be decoupled choosing the appropriate form of the potentials $\Gamma$ and $\tau$. Moreover, if we simultaneously make the transformations

$$
\Gamma \rightarrow \Gamma^{\prime}=\Gamma+\nabla \Lambda
$$

and

$$
\tau \rightarrow \tau^{\prime}=\tau-\frac{\partial \Lambda}{\partial t}
$$

in (5) and (8), we get the same original fields $\boldsymbol{g}$ and $\boldsymbol{K}$. Here, $\Lambda=\Lambda(x, y, z, t)$ is an arbitrary scalar function. We can choose this function in order to impose an additional condition over $\Gamma$ and $\tau$, in a similar way like the Lorentz or Coulomb gauge in the electromagnetic field, namely,

$$
\nabla \cdot \Gamma=0 \text { or } \nabla \cdot \Gamma=-\frac{1}{c^{2}} \frac{\partial \tau}{\partial t},
$$

which allows us to separate Equations (9) and (10) for the potentials $\tau$ and $\Gamma$. These potentials depend on the gauge condition we chose.

\section{Jefimenko's Equations for the Solenoidal and Irrotational Components}

Following the ideas of the work of Chubykalo et al. [9]-[11] and using the analogy be- 
tween the Maxwell's equations and the Jefimenko's ones [1] [2], we will apply the Helmholtz's theorem to define potentials that are independent of gauge transformations.

The Helmholtz's theorem claims that under certain conditions all vector fields can be represented as the sum of an irrotational and a solenoidal components. We will use this theorem to separate the fields $\boldsymbol{g}$ and $\boldsymbol{K}$.

Therefore, here we state the Helmholtz's theorem as [12]:

If the divergence $D(\boldsymbol{r})$ and a curl $\boldsymbol{C}(\boldsymbol{r})$ of a vector function $\boldsymbol{F}(\boldsymbol{r})$ are specified, and if they both go to zero faster than $1 / r^{2}$ as $r \rightarrow \infty$, and if $\boldsymbol{F}(\boldsymbol{r})$ itself tends to zero as $r \rightarrow \infty$, then $\boldsymbol{F}(\boldsymbol{r})$ is uniquely given by

$$
\boldsymbol{F}=-\nabla U+\nabla \times \boldsymbol{W},
$$

where

$$
U(\boldsymbol{r})=\frac{1}{4 \pi} \iiint_{\text {All space }} \frac{D\left(\boldsymbol{r}^{\prime}\right)}{\left|\boldsymbol{r}-\boldsymbol{r}^{\prime}\right|} \mathrm{d}^{3} \boldsymbol{r}^{\prime}
$$

and

$$
\boldsymbol{W}(\boldsymbol{r})=\frac{1}{4 \pi} \iiint_{\text {All space }} \frac{\boldsymbol{C}(\boldsymbol{r})}{\left|\boldsymbol{r}-\boldsymbol{r}^{\prime}\right|} \mathrm{d}^{3} \boldsymbol{r}^{\prime} .
$$

We are going to suppose that all conditions of this theorem are satisfied by the fields $\boldsymbol{g}$ and $\boldsymbol{K}$ defined by Equations (1) to $(4)^{1}$, and then, we apply Helmholtz's theorem to these quantities, including $J$. Thus, we obtain

$$
\begin{aligned}
\boldsymbol{g} & =\boldsymbol{g}_{i}+\boldsymbol{g}_{s}, \\
\boldsymbol{K} & =\boldsymbol{K}_{i}+\boldsymbol{K}_{s}, \\
\boldsymbol{J} & =\boldsymbol{J}_{i}+\boldsymbol{J}_{s},
\end{aligned}
$$

where the indices "i" and " $\mathrm{s}$ " mean irrotational and solenoidal components of the vectors, respectively.

For example:

$$
\begin{gathered}
\boldsymbol{J}_{i}=-\frac{1}{4 \pi} \nabla \iiint_{\text {All space }} \frac{\nabla^{\prime} \cdot \boldsymbol{J}}{\left|\boldsymbol{r}-\boldsymbol{r}^{\prime}\right|} \mathrm{d}^{3} \boldsymbol{r}^{\prime}, \\
\boldsymbol{J}_{s}=\frac{1}{4 \pi} \nabla \times \iiint_{\text {All space }} \frac{\nabla^{\prime} \times \mathbf{J}}{\left|\boldsymbol{r}-\boldsymbol{r}^{\prime}\right|} \mathrm{d}^{3} \boldsymbol{r}^{\prime},
\end{gathered}
$$

We are going to substitute $\boldsymbol{g}, \boldsymbol{K}$ and $\boldsymbol{J}$ given by the Equations (17)-(19) into the Jefimenko's Equations (1)-(4) and then, we obtain for the irrotational part:

$$
\begin{aligned}
& \nabla \cdot \boldsymbol{g}_{i}=-4 \pi G \varrho, \\
& \frac{\partial \boldsymbol{g}_{i}}{\partial t}=4 \pi G \boldsymbol{J}_{i}, \\
& \nabla \cdot \boldsymbol{K}_{i}=0, \\
& \frac{\partial \boldsymbol{K}_{i}}{\partial t}=0,
\end{aligned}
$$

${ }^{1}$ For systems localized in a finite region of space, it is evident that the fields $\boldsymbol{g}$ y $K$ depend on $r$ as $1 / r^{2}$. 
and the next equations for the solenoidal part:

$$
\begin{aligned}
& \nabla \times \boldsymbol{g}_{s}=-\frac{\partial \boldsymbol{K}_{s}}{\partial t}, \\
& \nabla \times \boldsymbol{K}_{s}-\frac{1}{c^{2}} \frac{\partial \boldsymbol{g}_{s}}{\partial t}=-\frac{4 \pi G}{c^{2}} \boldsymbol{J}_{s} .
\end{aligned}
$$

\section{The Gravitodynamical Potentials from Helmholtz's Theorem}

By definition, for the irrotational component of the gravitational field $\boldsymbol{g}_{i}$ we can define the scalar potential $\mathrm{T}$ as

$$
\nabla \mathrm{T}=-\boldsymbol{g}_{i}
$$

and if we substitute this relation into Equation (22), we obtain the Poisson's equation

$$
\nabla^{2} \mathrm{~T}=4 \pi G \varrho .
$$

Apparently, we need to take into account that $\mathrm{T}$ is not completely defined only by the Poisson's Equation (29), because we have another differential equation for $\mathrm{T}$, which can be obtained by substituting (28) into Equation (23)

$$
\frac{\partial}{\partial t} \nabla \mathrm{T}=-4 \pi G \boldsymbol{J}_{i}
$$

We show now that Equation (30) is equivalent to the law of conservation of mass. Indeed, let us take the divergence of the Equation (23), then we obtain as the result

$$
\frac{\partial}{\partial t}\left(\nabla \cdot \boldsymbol{g}_{i}\right)=4 \pi G \nabla \cdot \boldsymbol{J}_{i} .
$$

But from Equation (22) and because $\nabla \cdot \boldsymbol{J}_{i}=\nabla \cdot\left(\boldsymbol{J}_{i}+\boldsymbol{J}_{s}\right)=\nabla \cdot \boldsymbol{J}$, Equation (31) becomes the conservation mass law or the continuity equation

$$
\nabla \cdot \boldsymbol{J}+\frac{\partial \varrho}{\partial t}=0 .
$$

Now, we will demonstrate that the solution of Equation (30), indeed, is the same solution of the Poisson's Equation (29). To do this, we note that the irrotational component of $\boldsymbol{J}$ can be written as

$$
\boldsymbol{J}_{i}=-\nabla \phi_{J},
$$

where the potential $\phi_{J}$ is defined as

$$
\phi_{J}(x, y, z, t)=\frac{1}{4 \pi} \iiint_{\text {All space }} \frac{\nabla^{\prime} \cdot \boldsymbol{J}_{i}}{\boldsymbol{r}-\boldsymbol{r}^{\prime} \mid} \mathrm{d}^{3} \boldsymbol{r}^{\prime}
$$

or

$$
\phi_{J}(x, y, z, t)=-\frac{1}{4 \pi} \iiint_{\text {All space }} \frac{\frac{\partial}{\partial t} \varrho\left(\boldsymbol{r}^{\prime}, t\right)}{\left|\boldsymbol{r}-\boldsymbol{r}^{\prime}\right|} \mathrm{d}^{3} \boldsymbol{r}^{\prime},
$$

and where, if we relate Equations (32), (23), (28) y (33) and the fact that $\nabla \cdot \boldsymbol{J}_{i}=\nabla \cdot \boldsymbol{J}$, we have 


$$
\frac{\partial(-\nabla \mathrm{T})}{\partial t}=4 \pi G\left(-\nabla \phi_{J}\right) \Rightarrow \frac{\partial \mathrm{T}}{\partial t}=4 \pi G \phi_{J} .
$$

And from (36) and (34), we obtain

$$
\frac{\partial \mathrm{T}}{\partial t}=-G \iiint_{\text {All space }} \frac{\frac{\partial}{\partial t} \varrho\left(\boldsymbol{r}^{\prime}, t\right)}{\left|\boldsymbol{r}-\boldsymbol{r}^{\prime}\right|} \mathrm{d}^{3} \boldsymbol{r}^{\prime}
$$

or

$$
\mathrm{T}=-G \iiint_{\text {All space }} \frac{\varrho\left(\boldsymbol{r}^{\prime}, t\right)}{\left|\boldsymbol{r}-\boldsymbol{r}^{\prime}\right|} \mathrm{d}^{3} \boldsymbol{r}^{\prime},
$$

which is the solution of the Poisson's Equation (29). So we have found that the Poisson's equation given by Equation (29), completely defines the potential $\mathrm{T}$, together with its boundary conditions.

Since by definition $\boldsymbol{K}=\boldsymbol{K}_{s}$, then Equations (24) and (25) have the trivial solution $\boldsymbol{K}_{i}=0$.

Let us now apply the Helmholtz's theorem to the vector potential $\Gamma$. The Helmholtz theorem it is also known as the fundamental theorem of vector calculus (see Section III), and allows us to decompose every vectorial field in two components, an irrotational and a solenoidal one. Intuitively, it says that every vector function can be written as the sum of a divergence-free function (like $\Gamma_{s}$ ) and a curl-free function (like $\Gamma_{i}$ ), so that there exist scalar and vector potentials. So that $\Gamma=\Gamma_{i}+\Gamma_{s}$, because this is the form in which we can easily solve the system formed by Equations (26) and (27). Supposing that

$$
\boldsymbol{K}_{s}=\nabla \times \Gamma_{s}
$$

and taking into account (26) we obtain

$$
\boldsymbol{g}_{s}=-\frac{\partial \Gamma_{s}}{\partial t}
$$

One can substitute Equations (39) and (40) into (27), and we obtain

$$
\nabla^{2} \Gamma_{s}-\frac{1}{c^{2}} \frac{\partial^{2} \Gamma_{s}}{\partial t^{2}}=\frac{4 \pi G}{c^{2}} \boldsymbol{J}_{s},
$$

where we used the vector identity $\nabla \times(\nabla \times \boldsymbol{V})=\nabla(\nabla \cdot \boldsymbol{V})-\nabla^{2} \boldsymbol{V}$, for any arbitrary vector $\boldsymbol{V}$.

We have found that system of Equations (1)-(4) reduces to Equations (29) and (41), applying the Helmholtz's theorem. Therefore, we obtain separated equations for vector and scalar potentials, namely,

$$
\nabla^{2} \mathrm{~T}=4 \pi G \varrho
$$

and

$$
\nabla^{2} \Gamma_{s}-\frac{1}{c^{2}} \frac{\partial^{2} \Gamma_{s}}{\partial t^{2}}=\frac{4 \pi G}{c^{2}} \boldsymbol{J}_{s}
$$




\section{Invariance of the Potentials $\Gamma_{s}$ and $\mathrm{T}$ under Gauge Transformations}

Now, we will show that the potentials $\Gamma_{s}$ and $\mathrm{T}$ (which are gravitodynamical countertypes of the electromagnetic potentials $\boldsymbol{A}_{s}$ and $\Phi$ from [9]) are invariant under gauge transformations (11) and (12), in common with the fields $g$ and $K$. This will be the most prominent property of these potentials.

If we apply the Helmholtz theorem to the gravitational and cogravitational fields in terms of the ordinary potentials given by (5) and (8) without taking into account any gauge condition, we have

$$
\begin{aligned}
& \boldsymbol{g}_{i}=-\nabla \tau-\frac{\partial \Gamma_{i}}{\partial t}, \\
& \boldsymbol{g}_{s}=-\frac{\partial \Gamma_{s}}{\partial t}, \\
& \boldsymbol{K}_{i}=0, \\
& \boldsymbol{K}_{s}=\nabla \times \Gamma_{s},
\end{aligned}
$$

then, by definition, $\Gamma_{i}$ is given by

$$
\Gamma_{i}=-\nabla \phi_{\Gamma},
$$

where $\phi_{\Gamma}$ is an scalar function. If we substitute (48) into (44) then

$$
\boldsymbol{g}_{i}=-\nabla \tau-\frac{\partial}{\partial t}\left(-\nabla \phi_{\Gamma}\right)=-\nabla \tau+\nabla \frac{\partial \phi_{\Gamma}}{\partial t}=-\nabla\left(\tau-\frac{\partial \phi_{\Gamma}}{\partial t}\right),
$$

and from Equations (49) and (22) we have the relation between $\mathrm{T}$ and $\tau$.

$$
\mathrm{T}=\tau-\frac{\partial \phi_{\Gamma}}{\partial t} .
$$

Now, we apply the Helmholtz theorem and the gauge transformations (11) and (12) and from

$$
\Gamma^{\prime}=\Gamma_{i}^{\prime}+\Gamma_{s}^{\prime}=\Gamma_{i}+\Gamma_{s}+\nabla \Lambda,
$$

comparing the solenoidal parts we obtain

$$
\Gamma_{s}^{\prime}=\Gamma_{s} .
$$

If we seek the transformation law for $\phi_{\Gamma}$, then we can obtain the other transformation law for $\mathrm{T}$.

From Equation (51), we have the irrotational part of $\Gamma^{\prime}$

$$
\Gamma_{i}^{\prime}=\Gamma_{i}+\nabla \Lambda,
$$

and including Equation (48) in (53) we get

$$
-\nabla \phi_{\Gamma}^{\prime}=-\nabla \phi_{\Gamma}+\nabla \Lambda=-\nabla\left(\phi_{\Gamma}-\Lambda\right)
$$

or

$$
\phi_{\Gamma}^{\prime}=\phi_{\Gamma}-\Lambda
$$

At last, we can use Equation (50) for $\mathrm{T}$ and, if we consider Equations (11), (12) and 
(55), we obtain

$$
\mathrm{T}^{\prime}=\tau^{\prime}-\frac{\partial \phi_{\Gamma}^{\prime}}{\partial t}=\left(\tau-\frac{\partial \Lambda}{\partial t}\right)-\frac{\partial}{\partial t}\left(\phi_{\Gamma}-\Lambda\right)=\tau-\frac{\partial \phi_{\Gamma}}{\partial t}=\mathrm{T}
$$

Hence, we have checked that $\Gamma_{s}$ and $\mathrm{T}$ are invariants under gauge transformation and we can see that any gauge transformation is irrelevant if we use the Helmholtz's theorem.

It is convenient to remark that the fields $g$ and $K$ are generated only by $\Gamma_{s}$ and $\mathrm{T}$ given by (28), (39) and (40), so we can consider $\Gamma_{s}$ and $\mathrm{T}$ as the potentials generating the gravitodynamical field $\boldsymbol{g}$ and $\boldsymbol{K}$.

\section{Conclusions}

And so, we have shown that it is possible to define vector as well as scalar gravitodynamical potentials, which are invariant under gauge transformation. These potentials are defined uniquely from their differential Equations (42) and (43). For this reason, we have arguments for supposing the physical reality of these potentials, similarly to the fields $\boldsymbol{g}$ and $\boldsymbol{K}$ and unlike the gravitational potentials introduced by Jefimenko in [1], which are only used as a mathematical tool for calculating gravitational and cogravitational fields.

Our scalar potential $\mathrm{T}$ is a generator of the so-called instantaneous action at a distance in gravitation, and the vector potential $\Gamma_{s}$ can propagate with the velocity of light and it is responsible for the retarded action of the gravitodynamical field. So, one can conclude that retarded interaction in gravitodynamics takes place not instead but together with instantaneous action at a distance.

\section{References}

[1] Jefimenko, O.D. (2006) Gravitation and Cogravitation. Electret Scientific Company, Star City.

[2] Jefimenko, O.D. (2000) Causality Electromagnetic Induction and Gravitation: A Different Approach to the Theory of Electromagnetic and Gravitational Fields. 2nd Edition, Electret Scientific Company, Star City.

[3] Heaviside, O. (1893) The Electrician, 31 281-282.

[4] Brillouin, L. (1970) Relativity Reexamined. Academic Press, New York.

[5] Einstein, A. (1912) Vierteljahrschrift für gerichtliche Medizin und öffentliches Sanitatswessen, 44, 1-4.

[6] González, W.S. (2013) Gravitoelectromagnetismo y principio de Mach, eWT Ediciones.

[7] Vetö, B. (2010) European Journal of Physics, 31, 5.

[8] Vetö, B. (2011) European Journal of Physics, 32, 5.

[9] Chubykalo, A., Espinoza, A. and Alvarado Flores, R. (2011) Physica Scripta, 84, 015009-16. http://dx.doi.org/10.1088/0031-8949/84/01/015009

[10] Chubykalo, A., Espinoza, A. and Alvarado Flores, R. (2012) Physica Scripta, 85, 047002. http://dx.doi.org/10.1088/0031-8949/84/6/069502

[11] Chubykalo, A., Espinoza, A., Alvarado Flores, R. and Gutiérrez Rodríguez, A. (2006) 
Foundations of Physics Letteres, 19, 37-49.

http://dx.doi.org/10.1088/0031-8949/85/04/047002

[12] Arfken, G.B. and Weber, H.J. (1995) Mathematical Methods for Physicists. Academic Press, New York.

Submit or recommend next manuscript to SCIRP and we will provide best service for you:

Accepting pre-submission inquiries through Email, Facebook, LinkedIn, Twitter, etc. A wide selection of journals (inclusive of 9 subjects, more than 200 journals)

Providing 24-hour high-quality service

User-friendly online submission system

Fair and swift peer-review system

Efficient typesetting and proofreading procedure

Display of the result of downloads and visits, as well as the number of cited articles Maximum dissemination of your research work

Submit your manuscript at: http://papersubmission.scirp.org/ 